\title{
Sobrevida de prótese parcial fixa posterior em zircônia: revisão sistemática de estudos clínicos com até 7 anos de acompanhamento
}

\section{(Survival of zirconia-based posterior fixed partial prostheses: a systematic review of up to 7-year clinical follow-up studies)}

\author{
P. L. P. da Silva', G. A. Lemos', R. F. Bonan, J. R. C. Queiroz', A. U. D. Batista ${ }^{5}$ \\ ${ }^{1}$ Programa de Pós-graduação em Odontologia, Universidade Federal da Paraíba - UFPB, Centro de Ciências \\ da Saúde (CCS), Cidade Universitária, João Pessoa, PB 58051-900 \\ ${ }^{2}$ Instituto de Biologia, Universidade Estadual de Campinas - Unicamp, Departamento de Biologia Estrutural e \\ Funcional, Av. Bertrand Russel s/n, Campinas, SP 13083-865 \\ ${ }_{3}^{3}$ Programa de Pós-graduação em Odontologia, Universidade Federal de Pernambuco - UFPE, Av. Prof. Moraes \\ Rego, 1235, Cidade Universitária, Recife, PE 50670-901 \\ ${ }^{4}$ Curso de Odontologia, Universidade Estadual da Paraíba - UEPB, Rua Baraúnas, 351, Bairro Universitário, \\ Campina Grande, PB 58429-500 \\ ${ }^{5}$ Departamento de Odontologia Restauradora - UFPB, CCS, Cidade Universitária, João Pessoa, PB 58051-900 \\ pamelapan_lopes@hotmail.com,lemos.george@yahoo.com.br,robertabonan@gmail.com,joserenatocq@, \\ hotmail.com,andreulisses@yahoo.com.br
}

\begin{abstract}
Resumo
A cerâmica odontológica tem sofrido modificações na composição visando melhorar sua resistência em resistir às forças mastigatórias fisiológicas na região posterior da boca. O objetivo deste trabalho foi avaliar o comportamento clínico de próteses parciais fixas posteriores (PPFs) em zircônia a partir de estudos clínicos prospectivos de 3 anos ou mais. Realizou-se revisão sistemática da literatura por meio de pesquisa na base de dados PubMed em busca de publicações em inglês entre os anos de 2004 e 2014 com os seguintes descritores e suas combinações: 'fixed partial denture' OR 'dental prosthesis' OR 'dental porcelain' OR 'FPD' AND 'zirconia'. A busca resultou em 282 artigos, 20 foram lidos na íntegra e então oito selecionados. Os sistemas de zircônia utilizados foram Lava (3M ESPE), Cercon (DeguDent) e Denzir (Cad.esthetics) com cimentação variando entre resinosa, com ionômero de vidro e fosfato de zinco. A quantidade total de pacientes examinados foi de 199 , com 241 próteses instaladas, avaliados em um período de 3 a 7 anos. Destas, oito apresentaram fratura da infraestrutura em zircônia, 41 sofreram fratura apenas da cerâmica de recobrimento e 17 tiveram perda de retenção sem fratura; 17 dentes pilares sofreram cárie secundária, três fraturaram e nove necessitaram de tratamento endodôntico. A taxa de sobrevida das PPFs variou de 73,9\% a $100 \%$. Concluiu-se que a zircônia está apta a ser utilizada na região posterior, porém estudos são necessários para averiguar as causas das lascas do revestimento. Mediante as falhas na cimentação, recomenda-se seguir rigorosamente as instruções do fabricante e atentar para um preparo dental adequado.

Palavras-chave: prótese parcial fixa, cerâmica, sobrevida, revisão.
\end{abstract}

Abstract

\begin{abstract}
Dental ceramics has undergone changes in the composition to improve its strength and potential to withstand physiologic occlusal forces applied in the posterior region. The purpose of this work was to evaluate the clinical behavior of zirconia posterior fixed partial dentures (FPDs) from the analysis of prospective clinical studies with a minimum three-years observational period. A systematic review of literature was performed by searching the PubMed database for English-language publications, published from 2004 to 2014, using the following keywords and their combinations: 'fixed partial denture' OR 'dental prosthesis' OR 'dental porcelain' OR 'FPD' AND 'zirconia'. The search resulted in 282 articles, 20 full-text were analyzed and eight articles were selected for revision. Zirconia systems used were Lava (3M ESPE), Cercon (DeguDent), and Denzir (Cad.esthetics), cemented with resinous, glass ionomer and zinc-phosphate oxide cement. A total of 199 patients (241 prostheses) were examined, though a 3-7 years period. Of these FPD, eight had fracture of the zirconia framework, veneering ceramic fracture occurred in 41 FPD and 17 had loss of retention and debonding; 17 abutment teeth developed secondary caries, 3 fractured and nine required endodontic treatment. The survival rate of FPDs ranged from $73.9 \%$ to $100 \%$. In conclusion, zirconia is an adequate material for use in the posterior region, but more studies are needed for fully understand the reason for chipping of the veneering porcelain. To avoid cementation failures, it is recommended to strictly follow the manufacturer's instructions and pay attention to proper dental preparation.
\end{abstract}

Keywords: fixed partial denture, ceramics, survival, review. 


\section{INTRODUÇÃO}

A prótese parcial fixa (PPF) é utilizada com frequência na clínica odontológica, seja para solucionar a perda de um único dente ou na reabilitação de toda a oclusão. A substituição de dentes ausentes contribui para o maior conforto do paciente, com melhorias em sua capacidade de mastigação e na saúde e integridade dos arcos dentais, auxiliando sobremaneira no aumento da autoestima [1-3]. A crescente procura da sociedade pelo belo e harmonioso tem refletido também no dia-a-dia do cirurgião-dentista, onde as restaurações cerâmicas livres de metal têm se tornado a principal alternativa de tratamento para a reconstrução das estruturas dentárias perdidas [1-8]. Essa ampla utilização deve-se à capacidade das cerâmicas de mimetizar o dente natural, reproduzindo as propriedades ópticas do esmalte e da dentina, bem como sua textura; além disso, sua biocompatibilidade contribui para a manutenção da saúde periodontal e pulpar. As coroas metalo-cerâmicas, embora ainda largamente utilizadas, apresentam problemas estéticos, particularmente na margem cervical, uma vez que as ligas metálicas empregadas como infraestrutura interferem no fenômeno de reflexão e causam pigmentação dos tecidos pelo processo de corrosão [1-5, 7-9]. Por outro lado, a cerâmica constitui-se num material com pouca capacidade de sofrer deformação plástica haja vista sua alta friabilidade. Com o intuito de conferir-lhe resistência às forças mastigatórias e assim ampliar suas indicações - de coroas unitárias anteriores para PPFs em região posterior - houve o desenvolvimento de cerâmicas acrescidas de oxidos em sua composição básica, como alumina, leucita e zircônia [3-5, 7, 9, 10]. A escolha do sistema cerâmico a ser empregado não deve ser baseada na análise de uma propriedade mecânica apenas, mas sim numa avaliação comparativa de um conjunto delas - como resistência à flexão e à tração - aliado a estudos clínicos, pois fatores diversos influenciam a resistência mecânica e a longevidade das restaurações, diferindo em muitos aspectos das situações laboratoriais. Geralmente o método in vitro não é capaz de submeter materiais às mesmas condições que comportam in vivo, fazendo com que, muitas vezes, as propriedades de um material sejam superestimadas $[3,4,6$, 7, 11].

A resistência à fratura das coroas cerâmicas está baseada em diversos fatores, tais como obediência à indicação de cada tipo cerâmico de acordo com a região a ser reabilitada, preparo dental adequado, minuciosa execução laboratorial, espessura adequada do coping, tipo de cimentação e cuidadoso ajuste oclusal $[3,4,6,9,11]$. Ainda é necessário largo aprimoramento destes materiais no sentido de promover dureza compatível, com valores de resistência que permitam a utilização segura e duradoura das próteses ceramo-cerâmicas em áreas de esforço mastigatório, inclusive pontes fixas de maior extensão. Desta forma, tornase de suma importância seu avanço físico-químico, por meio de pesquisas clínicas e laboratoriais com o objetivo de aliar as propriedades estéticas, tão consagradas das porcelanas odontológicas, com valores de resistência que assegurem sua longevidade $[4,6,11]$. Assim, este artigo se propôs a avaliar o sucesso de três anos ou mais de próteses parciais fixas em zircônia na região posterior a partir da análise de estudos clínicos prospectivos, apontando as principais falhas técnicas e biológicas que levaram ao insucesso das próteses instaladas.

\section{MATERIAL E MÉTODOS}

Dois pesquisadores independentes realizaram pesquisa eletrônica na base de dados PubMed (Public Medline), vinculado ao MEDLINE (Medical Literature Analysis and Retrieval System Online) em busca de publicações em inglês entre os anos de 2004 e 2014, incluindo estudos clínicos prospectivos de próteses parciais fixas posteriores com infraestrutura em zircônia. Os seguintes descritores em inglês e suas combinações foram utilizados: 'fixed partial denture' OR 'dental prosthesis' OR 'dental porcelain' OR 'FPD' AND 'zirconia'. Os títulos e resumos identificados na busca inicial foram avaliados obedecendo rigorosamente aos critérios de inclusão e exclusão pré-estabelecidos. Os artigos selecionados e os artigos cujo título e resumo não eram esclarecedores foram obtidos na íntegra e reexaminados pelos dois pesquisadores de forma conjunta, a fim de elencar aqueles que fariam parte deste estudo. Foram incluídos ensaios clínicos randomizados e estudos prospectivos de próteses parciais fixas posteriores de três ou mais elementos e com pelo menos três anos de acompanhamento clínico em intervalos regulares. Os dados sobre a sobrevivência e a falha das PPFs instaladas deveriam ser relatados, bem como a descrição das complicações mais importantes. Foram excluídos estudos retrospectivos, transversais e casos clínicos. Estudos envolvendo coroas unitárias, próteses implanto-suportadas, inlay/onlay e estudos in vitro ou em modelos animais também foram excluídos.

Após leitura criteriosa dos artigos selecionados, os dados foram extraídos através de um formulário padronizado, permitindo o registro dos seguintes parâmetros: autor e ano de publicação, desenho do estudo e aspectos metodológicos, idade média dos pacientes avaliados, tempo de duração do estudo, intervalo entre as avaliações, número e localização das PPFs instaladas, tipo de sistema de zircônia utilizado, tipo de cerâmica de revestimento, tipo de cimentação e principais falhas e complicações apresentadas. As falhas e complicações registradas foram classificadas em falhas técnicas e biológicas [12]. Fratura da subestrutura de zircônia, fratura da cerâmica de revestimento, perda de retenção, comprometimento estético e descoloração das coroas e pônticos foram incluídas nas complicações técnicas. Presença de cárie secundária, necessidade de tratamento ou retratamento endodôntico, fratura e comprometimento periodontal dos dentes pilares foram considerados como complicações biológicas.

\section{RESULTADOS}

A pesquisa na base de dados empregando os descritores 
Tabela I - Caracterização dos estudos: tamanho da amostra, localização dos pônticos, tempo de avaliação, taxa de sobrevida, tipo de sistema de zircônia, cerâmica de recobrimento, cimento utilizado e conclusões.

[Table I - Studies characterization: sample size, location of the pontics, evaluation time, survival rate, type of zirconia system, veneering ceramic, cement used and conclusions.]

\begin{tabular}{|c|c|c|c|c|c|c|c|c|}
\hline $\begin{array}{l}\text { Artigo } \\
\text { e ano }\end{array}$ & Amostra & $\begin{array}{l}\text { Localização } \\
\text { dos pônticos }\end{array}$ & $\begin{array}{l}\text { Tempo de } \\
\text { avaliação }\end{array}$ & $\begin{array}{c}\text { Taxa de } \\
\text { sobrevida }\end{array}$ & $\begin{array}{l}\text { Sistema de } \\
\text { zircônia }\end{array}$ & $\begin{array}{l}\text { Cerâmica de } \\
\text { recobrimento }\end{array}$ & $\begin{array}{l}\text { Tipo de } \\
\text { cimento }\end{array}$ & Conclusões \\
\hline $\begin{array}{l}{[16]} \\
2013\end{array}$ & $\begin{array}{c}75 \\
\text { pacientes } \\
99 \text { PPFs }\end{array}$ & $\begin{array}{l}14 ; 15 ; 16 ; \\
24 ; 25 ; 26 ; \\
34 ; 35 ; 36 ; \\
45 ; 46 ; 47\end{array}$ & 7 anos & $93,8 \%$ & $\begin{array}{c}\text { Cercon } \\
\text { (DeguDent) }\end{array}$ & $\begin{array}{l}\text { Cercon Ceram } \\
S \text { (DeguDent) } \\
\text { e um material } \\
\text { experimental } \\
\quad(n=51)\end{array}$ & $\begin{array}{l}\text { Fosfato de } \\
\text { zinco } \\
\text { (Harvard, } \\
\text { Richter e } \\
\text { Hoffmann) }\end{array}$ & $\begin{array}{l}\text { PPFs posteriores } \\
\text { em zircônia tiveram } \\
\text { sobrevida menor que } \\
\text { a relatada para } \\
\text { metalo-cerâmicas. } \\
\text { Ocorreu fraturada } \\
\text { zircônia e do } \\
\text { recobrimento e } \\
\text { perda de retenção. }\end{array}$ \\
\hline $\begin{array}{l}{[13]} \\
2012\end{array}$ & $\begin{array}{c}17 \\
\text { pacientes } \\
20 \text { PPFs }\end{array}$ & $\begin{array}{c}15 ; 16 ; 25 \\
26 ; 35 ; 36 \\
45 ; 46\end{array}$ & 3 anos & $95 \%$ & $\begin{array}{c}\text { Lava } \\
\text { (3M ESPE) }\end{array}$ & $\begin{array}{l}\text { Lava Ceram } \\
(3 \mathrm{M} \text { ESPE) }\end{array}$ & $\begin{array}{l}\text { Resinoso } \\
\text { (Rely X } \\
\text { Unicem, } \\
\text { 3M ESPE) }\end{array}$ & $\begin{array}{c}\text { A zircônia é um } \\
\text { material confiável } \\
\text { para ser empregado } \\
\text { em PPFs posteriores. }\end{array}$ \\
\hline $\begin{array}{l}{[14]} \\
2012\end{array}$ & $\begin{array}{c}17 \\
\text { pacientes } \\
20 \text { PPFS }\end{array}$ & $\begin{array}{c}15 ; 16 ; 25 ; \\
26 ; 35 ; 36 \\
45 ; 46\end{array}$ & 4 anos & $95 \%$ & $\begin{array}{c}\text { Lava } \\
\text { (3M ESPE) }\end{array}$ & $\begin{array}{l}\text { Lava Ceram } \\
\text { (3M ESPE) }\end{array}$ & $\begin{array}{l}\text { Resinoso } \\
\text { (Rely X } \\
\text { Unicem, } \\
\text { 3M ESPE) }\end{array}$ & $\begin{array}{l}\text { PPFs em zircônia } \\
\text { demonstraram } \\
\text { sobrevida semelhante } \\
\text { às metalo-cerâmicas. }\end{array}$ \\
\hline $\begin{array}{l}{[17]} \\
2012\end{array}$ & $\begin{array}{c}25 \\
\text { pacientes } \\
25 \text { PPFs }\end{array}$ & $\begin{array}{c}15 ; 16 ; 24 ; \\
25 ; 26 ; 27 ; \\
35 ; 36 ; 37 \\
45 ; 46\end{array}$ & 5 anos & $92 \%$ & $\begin{array}{c}\text { Lava } \\
(3 \mathrm{M} \text { ESPE })\end{array}$ & $\begin{array}{l}\text { Lava Ceram } \\
\text { (3M ESPE) }\end{array}$ & $\begin{array}{l}\text { Ionômero } \\
\text { de vidro } \\
\text { (Ketac Cem, } \\
\text { 3M ESPE) }\end{array}$ & $\begin{array}{l}\text { A zircônia pode ser } \\
\text { utilizada em PPFs } \\
\text { posteriores. Maior fratura } \\
\text { do recobrimento tem } \\
\text { relação com desenho } \\
\text { inadequado ou bruxismo. }\end{array}$ \\
\hline $\begin{array}{l}{[15]} \\
2010\end{array}$ & $\begin{array}{c}75 \\
\text { pacientes } \\
99 \text { PPFs }\end{array}$ & $\begin{array}{l}14 ; 15 ; 16 ; \\
24 ; 25 ; 26 ; \\
34 ; 35 ; 36 ; \\
45 ; 46 ; 47\end{array}$ & 4 anos & $94 \%$ & $\begin{array}{c}\text { Cercon } \\
\text { (DeguDent) }\end{array}$ & $\begin{array}{l}\text { Cercon Ceram } \\
S \text { (DeguDent) } \\
\text { e um material } \\
\text { experimental } \\
\quad(n=51)\end{array}$ & $\begin{array}{c}\text { Fosfato } \\
\text { de zinco } \\
\text { (Harvard, } \\
\text { Richter e } \\
\text { Hoffmann) }\end{array}$ & $\begin{array}{c}\text { A zircônia tem } \\
\text { sobrevida adequada } \\
\text { para ser utilizada } \\
\text { em PPFs posteriores. }\end{array}$ \\
\hline $\begin{array}{l}{[19]} \\
2009\end{array}$ & $\begin{array}{c}19 \\
\text { pacientes } \\
\text { 21 PPFs }\end{array}$ & $\begin{array}{l}\text { oito pré- } \\
\text { molares; doze } \\
1^{\circ} \text { molares e } \\
\text { um } 2^{\circ} \text { molar }\end{array}$ & 3 anos & $90,5 \%$ & $\begin{array}{c}\text { Cercon } \\
\text { (DeguDent) }\end{array}$ & $\begin{array}{c}\text { Cercon Ceram } \\
\text { Express } \\
\text { (DeguDent) }\end{array}$ & $\begin{array}{c}\text { Ionômero } \\
\text { de vidro } \\
\text { (Ketac Cem, } \\
\text { 3M ESPE) }\end{array}$ & $\begin{array}{c}\text { A zircônia exibiu } \\
\text { força suficiente para } \\
\text { ser utilizada em PPFs } \\
\text { posteriores de três } \\
\text { elementos. }\end{array}$ \\
\hline $\begin{array}{l}{[20]} \\
2008\end{array}$ & $\begin{array}{c}18 \\
\text { pacientes } \\
19 \text { PPFs }\end{array}$ & $\begin{array}{c}14 ; 16 ; 24 ; \\
25 ; 26 ; 34 ; \\
35 ; 36 ; 45 ; 46\end{array}$ & 5 anos & $100 \%$ & $\begin{array}{c}\text { Denzir } \\
\text { (Cad. } \\
\text { esthetics) }\end{array}$ & $\begin{array}{l}\text { Vita D } \\
\text { (Vita) }\end{array}$ & $\begin{array}{l}\text { Fosfato de } \\
\text { zinco (De } \\
\text { Trey Zinc, } \\
\text { Dentsply) } \\
\text { e Resinoso } \\
\text { (Panavia F, } \\
\text { Kuraray) }\end{array}$ & $\begin{array}{l}\text { É promissor o uso } \\
\text { da zircônia em PPFs } \\
\text { posteriores de até } \\
\text { três elementos. }\end{array}$ \\
\hline $\begin{array}{l}{[18]} \\
2007\end{array}$ & $\begin{array}{c}45 \\
\text { pacientes } \\
57 \text { PPFs }\end{array}$ & $\begin{array}{l}40 \text { elementos } \\
\text { superiores e } \\
17 \text { inferiores }\end{array}$ & 5 anos & $73,9 \%$ & $\begin{array}{c}\text { Cercon } \\
\text { (DeguDent) }\end{array}$ & $\begin{array}{c}\text { Cercon Ceram } \\
\text { Express } \\
\text { (DeguDent) }\end{array}$ & $\begin{array}{c}\text { Resinoso } \\
\text { (Panavia } \\
\text { TC, Kuraray } \\
\text { e Variolink, } \\
\text { Ivoclar) }\end{array}$ & $\begin{array}{l}\text { A zircônia pode ser } \\
\text { empregada em PPFs } \\
\text { posteriores de } 3 \text { e } 4 \\
\text { elementos. A cerâmica } \\
\text { de recobrimento } \\
\text { precisa ser melhorada. }\end{array}$ \\
\hline
\end{tabular}


Tabela II - Falhas técnicas e biológicas apontadas nos estudos - número de PPFs e elementos dentários acometidos. [Table II - Technical and biological failures identified in the studies - number of FPDs and affected teeth.]

\begin{tabular}{ccccccc}
\hline & \multicolumn{3}{c}{ Falhas técnicas em pônticos e coroas } & \multicolumn{3}{c}{ Falhas biológicas em dentes pilares } \\
Artigo e ano & $\begin{array}{c}\text { Fratura da } \\
\text { zircônia }\end{array}$ & $\begin{array}{c}\text { Fratura do } \\
\text { recobrimento }\end{array}$ & $\begin{array}{c}\text { Perda de } \\
\text { retenção }\end{array}$ & $\begin{array}{c}\text { Cárie } \\
\text { secundária }\end{array}$ & $\begin{array}{c}\text { Tratamento } \\
\text { endodôntico }\end{array}$ & Fratura \\
\hline$[16] 2013$ & 5 & 23 & 11 & 4 & 5 & 1 \\
{$[13] 2012$} & 0 & 2 & 0 & 0 & 0 & 1 \\
{$[14] 2012$} & 0 & 0 & 0 & 0 & 0 & 1 \\
{$[17] 2012$} & 1 & 5 & 0 & 0 & 1 & 0 \\
{$[15] 2010$} & 1 & 13 & 9 & 4 & 1 & 1 \\
{$[19] 2009$} & 1 & 4 & 2 & 3 & 2 & 0 \\
{$[20] 2008$} & 0 & 0 & 0 & 0 & 0 & 0 \\
{$[18] 2007$} & 1 & 7 & 4 & 10 & 1 & 1 \\
\hline
\end{tabular}

pré-estabelecidos resultou em 282 artigos. Após a leitura dos títulos e dos resumos, 20 trabalhos foram selecionados e lidos na íntegra. Destes, apenas oito foram escolhidos, pois obedeceram a todos os critérios de inclusão e exclusão. Os 12 artigos descartados analisaram coroas unitárias e/ou metalocerâmicas ou, ainda, não cumpriram os três anos mínimos de acompanhamento. Os trabalhos selecionados correspondem a pesquisas recentes publicadas nos últimos oito anos. Os sistemas de zircônia utilizados foram Lava (3M ESPE), Cercon (DeguDent) e Denzir (Cad.esthetics). A cerâmica de recobrimento empregada foi aquela correspondente a cada tipo de sistema de zircônia; já o tipo de cimentação variou entre resinosa, com ionômero de vidro e fosfato de zinco, conforme pode ser observado na Tabela I.

A quantidade de pacientes examinados em cada estudo oscilou entre 17 e 75 . Considerando que em [13, 14] foram avaliados os mesmos pacientes por um período de três e quatro anos, respectivamente, enquanto que em $[15,16]$ foram avaliados também os mesmos pacientes por um período de quatro e sete anos, tem-se um total de 199. Em alguns pacientes foi instalada mais de uma PPF, somando um número de 241 próteses instaladas, as quais foram acompanhadas clinicamente num período entre três e cinco anos, com visitas regulares ao cirurgião-dentista. A taxa de sobrevida das PPFs variou de 73,9\% a $100 \%$. Do total de próteses avaliadas, oito apresentaram fratura da infraestrutura em zircônia, enquanto que 41 sofreram fratura apenas da cerâmica de recobrimento e 17 tiveram perda de retenção sem fratura, como discriminado na Tabela II, onde se pode observar também que dentre os dentes pilares, 17 sofreram cárie secundária, três fraturaram e nove necessitaram de tratamento endodôntico.

\section{DISCUSSÃO}

Diante de sua alta capacidade de mimetizar a estrutura dental, a cerâmica vem sendo cada vez mais empregada na Odontologia; reconhecidamente um material friável, tem sofrido mudanças em sua composição e estrutura molecular para se obter um ganho na resistência às forças mastigatórias e assim ampliar seu uso. Se antes estava indicada apenas para coroas unitárias na região anterior da boca, agora, com o advento dos sistemas cerâmicos à base de zircônia, pode ser empregada em reabilitações maiores, contudo, a sobrevida clínica dessas próteses precisa ser mais bem avaliada [3-7, $10,11]$. As cerâmicas de uso odontológico são caracterizadas por uma fase cristalina a qual está circundada por uma fase vítrea. A proporção da cadeia de óxido de silício na matriz vítrea está relacionada com a viscosidade e a expansão térmica da cerâmica. Já suas propriedades mecânicas e ópticas são ditadas pela composição da fase cristalina [8]. Os trabalhos avaliados fizeram uso de diferentes sistemas - Cercon (Dentisply), Lava (3M ESPE) e Denzir (Cad. esthetics) - para confecção de peças ceramo-cerâmicas à base de zircônia, e cada um destes emprega tipos específicos de zircônia formados pela adição de diferentes elementos em sua composição [3, 6, 7].

Cercon (Dentisply) e Lava (3M ESPE) fabricam as peças através da usinagem utilizando-se da tecnologia CAD/CAM (Computer-aided design/Computer-aided manufacturing). O preparo do dente é escaneado diretamente na boca ou a partir de modelos de gesso; a imagem obtida é processada e a restauração é executada por meio da fresagem de blocos de cerâmica pré-sinterizados seguindo o desenho planejado. Para compensar a contração que ocorre após a queima para sinterização final, os blocos são fresados em tamanho 20 a $30 \%$ maior do que o trabalho original [37]. Nesses sistemas a zircônia é conhecida como Y-TZP (cerâmica de zircônia tetragonal policristalina estabilizada com ítria); o óxido de ítrio adicionado na zircônia pura tem o objetivo de estabilizar a fase tetragonal na temperatura ambiente, gerando um material policristalino que devido à sua microestrutura não possui fases vítreas, evitando sua degradação ou desestabilização pela saliva e consequente aumento da propagação de trincas [4, 6-9]. Este tipo de zircônia apresenta algumas características mecânicas superiores aos outros sistemas cerâmicos, como maiores resistências à flexão e à fratura [8, 9], permitindo a confecção de conectores de menor área [8], o que acarreta um menor desgaste de estrutura dental. Três dos trabalhos 
avaliados [13, 14, 17] empregaram o Lava, obtendo integridade marginal excelente, com uma sobrevida de $95 \%$; os autores recomendam seu uso na região posterior da boca, mas atentam para a ocorrência de lascas no revestimento cerâmico. O sistema Cercon foi utilizado em outros quatro estudos [15, 16, 18, 19]. Em [18] foi obtida sobrevida mais baixa entre os trabalhos estudados, cerca de $76 \%$; os autores testaram uma cerâmica de recobrimento experimental e empregaram dois tipos de cimentação resinosa ocorrendo muitos problemas de selamento. Em [15] bem como em [16] foi utilizada a mesma cerâmica de recobrimento e também apresentaram a perda de retenção como um dos maiores problemas, mas com uma taxa de sobrevida maior e uso do cimento de fosfato de zinco. Em [19] foi obtida uma sobrevida relativamente baixa de $90,5 \%$, porém não relataram lascas no revestimento. Denzir (Cad. esthetics), testada em [20], tratase de uma zircônia parcialmente estabilizada por magnésia. Os autores empregaram a tecnologia CAD/CAM, obtendo uma sobrevida de 100\%; após um acompanhamento de cinco anos não houve nenhuma falha técnica ou biológica.

Os casos de fratura da subestrutura de zircônia, fratura da cerâmica de revestimento, perda de retenção, comprometimento estético e descoloração das coroas e pônticos foram incluídos nas complicações técnicas. Poucos artigos avaliaram o comprometimento estético, priorizando somente a resistência mecânica em suas avaliações. A estética foi considerada satisfatória [13, 14, 17-19], sendo mencionada apenas pequena mudança de cor marginal sem evidência de cárie [18]. A opacidade advinda da adição de óxido de zircônio não prejudicou a estética $[1-6,10,11]$. Foram raros os casos de fratura da infraestrutura em zircônia e os maiores problemas estiveram na fratura do recobrimento cerâmico; das 241 próteses instaladas, apenas oito sofreram fratura da infraestrutura de zircônia, enquanto que 41 sofreram lascas em seu revestimento. $\mathrm{O}$ fato das fraturas terem ocorrido em zircônias do tipo Y-TZP leva a crer que houve alguma falha pontual na técnica de processamento [12]. Apesar da ocorrência de lascas no revestimento na maioria dos casos não ocasionar a remoção das próteses, sendo solucionado apenas com polimento coronário, devese acompanhar a longo prazo afim de encontrar suas causas e tentar saná-las.

$\mathrm{Na}$ literatura deparam-se com algumas explicações para essa fragilidade do revestimento, tais como: falhas de adesão entre o recobrimento e a infraestrutura [1]; diferenças entre o coeficiente de expansão térmica; erros no processamento na interface zircônia/revestimento que podem transformar a fase tetragonal em monocíclica; presença de tensões residuais [12]; alta rugosidade superficial [13, 14]; paciente com bruxismo; e conformação anatômica inadequada do preparo dental e/ou da prótese [15]. Um outro quesito importante é a preocupação com o espaço disponível para reabilitação, permitindo uma espessura adequada do revestimento; alguns autores $[13,14,16]$ empregaram como critérios de inclusão uma área de pelo menos $9 \mathrm{~mm}$ de altura para confecção de conector capaz de suportar as cargas mastigatórias. Já em [19] foi preconizada uma área disponível para a coroa clínica de $5 \mathrm{~mm}$ ou mais de altura. A fratura da zircônia pode ter ocorrido devido ao efeito de cunha aliado à sua pequena espessura e localização desfavorável na região de conector [15], ou ainda à confecção de um preparo dental inadequado [15], conforme detalhado anteriormente. Os trabalhos [15, $16,18]$ obtiveram o maior número de lascas do revestimento bem como perda de retenção. Apesar da perda de retenção também não ocasionar a remoção definitiva da prótese, já que esta pode ser recimentada, deve-se ter atenção aos problemas advindos, como cáries secundárias. Sua causa deve estar em falhas na manipulação e não no tipo de cimento, uma vez que em [20] foi empregado o mesmo tipo de cimentação (cimento resinoso e fosfato de zinco), mas não se enfrentou esses problemas. Porém, em [16] percebeuse que o uso do fosfato de zinco levou a um aumento na taxa de perda de retenção e concluiu-se que o tipo de cimento parece sim ser um fator crítico a longo prazo. Não há uma explicação clara para a perda de retenção apontada nos estudos clínicos de seguimento. Não há ligação com o tipo de cimento, já que ocorre tanto nas próteses cimentadas pela técnica adesiva como pela técnica convencional, não havendo vantagem no uso de uma em detrimento da outra [12]. Contudo, uma cimentação confiável requer um correto tratamento de superfície, tanto do preparo dental quanto da prótese, seja através do uso de jateamentos, silicatização, silanos, primers específicos para zircônia e ácidos [9].

Cárie secundária, necessidade de tratamento ou retratamento endodôntico, fratura e comprometimento periodontal dos dentes pilares foram considerados como complicações biológicas. Nenhum estudo relatou comprometimento periodontal quando comparado com dentes hígidos, corroborando com a excelente biocompatibilidade da cerâmica aos tecidos orais mencionada na literatura como uma das vantagens deste tipo de material frente às próteses metalo-cerâmicas $[1,2$, 5, 11]. Foram relatadas cáries secundárias, sensibilidade e necessidade de tratamento endodôntico, e os autores encontraram relação entre fenda marginal e presença de cárie secundária $[16,18]$. Provavelmente ocorreu falha na manipulação dos materiais, ou, no caso do fosfato de zinco, solubilização do mesmo pela presença de falhas marginais. Entretanto, tais problemas não foram relatados por outros trabalhos e não houve diferença estatística significante entre os dois tipos de cimentos resinosos empregados. Também foi relatada necessidade de tratamento endodôntico devido a sensibilidade e fratura dental $[16,19]$. As falhas apontadas pelos estudos parecem não ter associação com o tipo de recobrimento empregado, extensão da PPF ou o arco de instalação na boca [16].

\section{CONCLUSÃO}

Após análises anuais por até cinco anos, as taxas de sobrevida das próteses instaladas alcançaram bons valores e a zircônia foi considerada apta a ser utilizada na região posterior, porém é importante a continuidade desses estudos para que o uso clínico da zircônia em períodos mais longos 
seja avaliado, frente às metalo-cerâmicas, que já tem seu uso consagrado. As principais falhas envolvidas no insucesso das próteses foram perda de retenção, lascas no revestimento cerâmico e presença de cárie secundária. Os problemas envolvendo a cimentação estiveram ligados não ao tipo de cimento empregado, mas provavelmente à falha em sua manipulação e/ou a preparos dentais inadequados. Recomenda-se a realização de estudos para averiguar as causas associadas ao lascamento do revestimento cerâmico.

\section{REFERÊNCIAS}

[1] S. Costa, R. Fraga, A. Martins, Odonto 19, 37 (2011) 33. [2] R.A. Panadero, J.L.R. Rodríguez, A. Ferreiroa, M. Ruíz, A.F. Font, J. Clin. Exp. Dent. 6, 1 (2014) 66.

[3] L. Garcia, S. Consani, P. Cruz, F. Souza, RGO 59, 0 (2011) 67.

[4] T. Oguri, Y. Tamaki, Y. Hotta, T. Miyazaki, Dent. Mater. J. 31, 5 (2012) 788.

[5] L. Peixoto, E. Akaki, Arq. Bras. Odontol. 4, 2 (2008) 96. [6] G. Aktas, E. Sahin, P. Vallittu, M. Özcan, L. Lassila, Int. J. Oral Sci. 5, 4 (2013) 236.

[7] T. Miyazaki, T. Nakamura, H. Matsumura, S. Ban, T. Kobayashi, J. Prosthodont. Res. 57, 4 (2013) 236.

[8] E. Gomes, W. Assunção, E. Rocha, P. Santos, Cerâmica
54, 331 (2008) 319.

[9] R. Andreiuolo, S. Gonçalves, K. Dias, Ver. Bras. Odontol. 68, 1 (2011) 49.

[10] R. Nishioka, L. Vasconcelos, K. Tanaka, G. Duarte Filho, R. Jóias, PCL 6, 34 (2004) 540.

[11] A. Bohjalian, E. Froner, A.L. Zanetti, V. Santos, RGO 54, 2 (2006) 185.

[12] J. Schley, N. Heussen, S. Reich, J. Fischer, K. Haselhuhn, S. Wolfart, Eur. J. Oral Sci. 118, 5 (2010) 443.

[13] J. Peláez, P. Cogolludo, B. Serrano, J. Lozano, M. Suárez, J. Prosthet. Dent. 107, 6 (2012) 373.

[14] J. Peláez, P. Cogolludo, B. Serrano, J. Lozano, M. Suárez, Int. J. Prosthodont. 25, 5 (2012) 451.

[15] M. Roediger, N. Gersdorff, A. Huels, S. Rinke, Int. J. Prosthodont. 23, 2 (2010) 141.

[16] S. Rinke, N. Gersdorff, K. Lange, M. Roediger. Int. J. Prosthodont. 26 (2013) 164.

[17] J. Schmitt, M. Goellner, U. Lohbauer, M. Wichmann, S. Reich, Int. J. Prosthodont. 25, 6 (2012) 585.

[18] I. Sailer, A. Fehér, F. Filser, L. Gauckler, H. Lüthy, C. Hämmerle, Int. J. Prosthodont. 20, 4 (2007) 383.

[19] F. Beur, D. Edelhoff, W. Gernet, J. Sorensen, Clin. Oral Investig. 13, 4 (2009) 445.

[20] M. Molin, S. Karlsson, Int. J. Prosthodont. 21 (2008) 223.

(Rec. 29/06/2015, Rev. 08/10/2015, Ac. 07/11/2015) 